\title{
Compressible laminar boundary-layer flows of a dusty gas over a semi-infinite flat plate
}

\author{
By B. Y. WANG \\ Institute of Mechanics, Academia Sinica, Beijing, China \\ AND I. I. GLASS \\ Institute for Aerospace Studies, University of Toronto, 4925 Dufferin Street, Downsview, \\ Ontario, Canada, M3H 5 T6
}

(Received 8 December 1986 and in revised form 15 June 1987)

The compressible laminar boundary-layer flows of a dilute gas-particle mixture over a semi-infinite flat plate are investigated analytically. The governing equations are presented in a general form where more reasonable relations for the two-phase interaction and the gas viscosity are included. The detailed flow structures of the gas and particle phases are given in three distinct regions: the large-slip region near the leading edge, the moderate-slip region and the small-slip region far downstream. The asymptotic solutions for the two limiting regions are obtained by using a seriesexpansion method. The finite-difference solutions along the whole length of the plate are obtained by using implicit four-point and six-point schemes. The results from these two methods are compared and very good agreement is achieved. The characteristic quantities of the boundary layer are calculated and the effects on the flow produced by the particles are discussed. It is found that in the case of laminar boundary-layer flows, the skin friction and wall heat-transfer are higher and the displacement thickness is lower than in the pure-gas case alone. The results indicate that the Stokes-interaction relation is reasonable qualitatively but not correct quantitatively and a relevant non-Stokes relation of the interaction between the two phases should be specified when the particle Reynolds number is higher than unity.

\section{Introduction}

Boundary-layer flows of a gas-particle mixture have practical applications in many scientific and technical fields, such as in solid rocket exhaust nozzles, nuclear reactors with gas-solid feeds, ablation cooling, blast waves moving over the Earth's surface, conveying of powdered materials, fluidized beds and environmental pollutants (Rudinger 1980). Incompressible laminar boundary-layer flows of a dilute gas-particle suspension were studied using several analytical methods: a series method (Marble 1963; Liu 1967; Soo 1968; Otterman \& Lee 1970; DiGiovanni \& Lee 1974), an integral method (Soo 1967; Tabakoff \& Hamed 1972; Jain \& Ghosh 1979) and a finite-difference method (Hamed \& Tabakoff 1973; Osiptsov 1980; Prabha \& Jain 1982). Singleton (1965) extended Marble's analysis (1963) to the compressible case where the density of the gas phase as well as the particle phase may change. $\mathrm{He}$ obtained the asymptotic solutions for the large- and small-slip regions. However, he developed the governing boundary-layer equations assuming that the viscosity-temperature relation has the special form $\mu^{*} / \mu_{\infty}^{*}=\left(T^{*} / T_{\infty}^{*}\right)^{\frac{1}{2}}$ and that the 


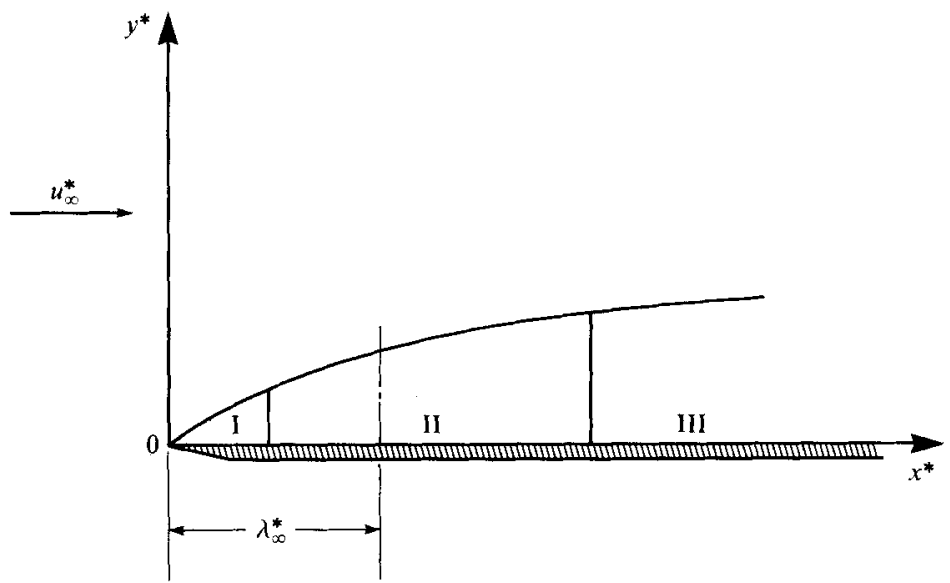

Figure 1. Geometry of the problem. Region I : large slip; region II : moderate slip; region III: small slip.

interaction between the two phases takes place through the Stokes relation. Moreover, no detailed information was available until the present analysis on the boundary-layer development in the non-equilibrium transition region, where the slip between the gas and particles is moderate.

In this paper, the behaviour of compressible laminar boundary-layer flows of a dilute dusty gas over a semi-infinite flat plate along the entire length of the plate is studied (see figure 1). The basic equations for the two phases are derived in a more general form. A series-expansion method, similar to Singleton's (1965), is employed to give the asymptotic solutions. The asymptotic results obtained in this research, however, are presented for the more realistic situation by using a power law for the variation of the gas viscosity with temperature and a non-Stokes relation for the twophase interaction. In order to study the boundary-layer flow over the whole length along the plate, an implicit finite-difference scheme, which combines a six-point scheme with four-point schemes, is constructed. The profiles of flow variables for the two phases are given at different distances from the leading edge to far downstream. The asymptotic large-slip solution provides the initial profiles of flow properties necessary to start the finite-difference procedure. In addition, the asymptotic solutions can be used to verify the correctness of the finite-difference method. Some characteristic quantities of the boundary layer including the skin friction coefficient, the heat-transfer rate at the wall and the displacement thickness, are calculated as functions of the distance along the plate. The alterations of the flow properties owing to the presence of particles and the non-Stokes relation are discussed in detail.

\section{Governing boundary-layer equations}

In this analysis, the gas-particle mixture is assumed as a dilute two-phase system where the volume fraction of the particles is neglected. Besides, there are no radiative heat transfer, chemical reactions, coagulation, phase change and deposition in this system. The gas phase is a perfect gas. The particle phase is treated as a continuum consisting of small solid spheres of uniform size. The particles have no individual random motion, mutual collisions and other interactions among them. Only the process of drag and heat transfer couple the particles with the gas. The drag 
coefficient and the Nusselt number for a single sphere in a viscous flow are assumed valid for the particle cloud.

With the above assumptions, the basic equations for steady, compressible, laminar, two-dimensional boundary-layer flows of dusty gases over a flat plate are given by the equations of mass, momentum, energy and state for the gas and particles,

$$
\begin{gathered}
\frac{\partial}{\partial x^{*}} \rho^{*} u^{*}+\frac{\partial}{\partial y^{*}} \rho^{*} v^{*}=0 \\
\rho^{*}\left(u^{*} \frac{\partial u^{*}}{\partial x^{*}}+v^{*} \frac{\partial u^{*}}{\partial y^{*}}\right)=\frac{\partial}{\partial y^{*}}\left(\mu^{*} \frac{\partial u^{*}}{\partial y^{*}}\right)+\rho_{\mathrm{p}}^{*} \frac{u_{\mathrm{p}}^{*}-u^{*}}{\tau_{\mathrm{v}}^{*}} D \\
c_{\mathrm{p}}^{*} \rho^{*}\left(u^{*} \frac{\partial T^{*}}{\partial x^{*}}+v^{*} \frac{\partial T^{*}}{\partial y^{*}}\right)=\frac{\partial}{\partial y^{*}}\left(k^{*} \frac{\partial T^{*}}{\partial y^{*}}\right)+\mu^{*}\left(\frac{\partial u^{*}}{\partial y^{*}}\right)^{2} \\
+\rho_{\mathrm{p}}^{*} \frac{\left(u_{\mathrm{p}}^{*}-u^{*}\right)^{2}+\left(v_{\mathrm{p}}^{*}-v^{*}\right)^{2}}{\tau_{\mathrm{v}}^{*}} D+c_{\mathrm{s}}^{*} \rho_{\mathrm{p}}^{*} \frac{T_{\mathrm{p}}^{*}-T^{*}}{2 \tau_{\mathrm{T}}^{*}} N u \\
p^{*}=\rho^{*} R^{*} T^{*} \\
\frac{\partial}{\partial x^{*}} \rho_{\mathrm{p}}^{*} u_{\mathrm{p}}^{*}+\frac{\partial}{\partial y^{*}} \rho_{\mathrm{p}}^{*} v_{\mathrm{p}}^{*}=0 \\
\rho_{\mathrm{p}}^{*}\left(u_{\mathrm{p}}^{*} \frac{\partial u_{\mathrm{p}}^{*}}{\partial x^{*}}+v_{\mathrm{p}}^{*} \frac{\partial u_{\mathrm{p}}^{*}}{\partial y^{*}}\right)=-\rho_{\mathrm{p}}^{*} \frac{\left(u_{\mathrm{p}}^{*}-u^{*}\right)}{\tau_{\mathrm{v}}^{*}} D \\
\rho_{\mathrm{p}}^{*}\left(u_{\mathrm{p}}^{*} \frac{\partial v_{\mathrm{p}}^{*}}{\partial x^{*}}+v_{\mathrm{p}}^{*} \frac{\partial v_{\mathrm{p}}^{*}}{\partial y^{*}}\right)=-\rho_{\mathrm{p}}^{*} \frac{\left(v_{\mathrm{p}}^{*}-v^{*}\right)}{\tau_{\mathrm{v}}^{*}} D \\
c_{\mathrm{s}}^{*} \rho_{\mathrm{p}}^{*}\left(u_{\mathrm{p}}^{*} \frac{\partial T_{\mathrm{p}}^{*}}{\partial x^{*}}+v_{\mathrm{p}}^{*} \frac{\partial T_{\mathrm{p}}^{*}}{\partial y^{*}}\right)=-c_{\mathrm{s}}^{*} \rho_{\mathrm{p}}^{*} \frac{T_{\mathrm{p}}^{*}-T^{*}}{2 \tau_{\mathrm{T}}^{*}} N u
\end{gathered}
$$

where $p, \rho, T, u, v$ represent the pressure, density, temperature, tangential velocity and normal velocity, respectively. The superscript * refers to dimensional parameters and the subscript $\mathrm{p}$ to the particle phase. Also, $R^{*}$ is the gas constant; $c_{\mathrm{p}}^{*}$ and $c_{\mathrm{s}}^{*}$ are the specific heats for the gas and particles: $\tau_{\mathrm{v}}^{*}=\rho_{\mathrm{s}}^{*} d^{* 2} / 18 \mu^{*}$ and $\tau_{\mathrm{T}}^{*}=\rho_{\mathrm{S}}^{*} d^{* 2} c_{\mathrm{s}}^{*} / 12 k^{*}$ are the velocity and temperature equilibrium times for the particles $\left(\rho_{\mathrm{s}}^{*}\right.$ is the density of the particle material and $d^{*}$ is the particle diameter). In order to close the basic equations, it is necessary to specify the dependence of $\mu^{*}, k^{*}, D$ and $N u$ on the flow variables. In the present paper, a power relation for the gas viscosity $\mu^{*}$ is employed:

$$
\frac{\mu^{*}}{\mu_{\infty}^{*}}=\left(\frac{T^{*}}{T_{\infty}^{*}}\right)^{\omega} \quad(0.5 \leqslant \omega \leqslant 1.0),
$$

where $\omega$ is the power index and the subscript $\infty$ refers to the free-stream value. As usual, the heat conductivity $k^{*}$ can be expressed as $k^{*}=c_{\mathrm{p}}^{*} \mu^{*} / \operatorname{Pr}$, where $\operatorname{Pr}$ is the gas Prandtl number. $D$ and $N u$ are the normalized drag coefficient based on Stokes drag coefficient and the Nusselt number based on the particle diameter. They depend on the interaction mechanism between the two phases. In the Stokes case, $D=1.0$ and $N u=2.0$. In the non-Stokes case, the following relations are chosen here according to Gilbert, Davis \& Altman (1955) and Knudsen \& Katz (1958),

$$
\begin{aligned}
D & =\frac{1}{50} R e_{\mathrm{s}}+\frac{7}{6} R e_{\mathrm{s}}^{0.15}, \\
N u & =2.0+0.6 \operatorname{Pr}^{\frac{1}{3}} \operatorname{Re}_{8}^{\frac{1}{2}},
\end{aligned}
$$


where $R e_{\mathrm{s}}$ is the slip Reynolds number of the particles:

$$
R e_{\mathrm{s}}=\frac{\rho^{*}\left[\left(u_{\mathrm{p}}^{*}-u^{*}\right)^{2}+\left(v_{\mathrm{p}}^{*}-v^{*}\right)^{2}\right]^{\frac{1}{2}} d^{*}}{\mu^{*}} .
$$

The boundary conditions for the problem under consideration are:

(i) At the wall of the flat plate,

$$
u^{*}\left(x^{*}, 0\right)=0, \quad v^{*}\left(x^{*}, 0\right)=0, \quad T^{*}\left(x^{*}, 0\right)=T_{\mathrm{w}}^{*}, \quad v_{\mathrm{p}}^{*}\left(x^{*}, 0\right)=0 .
$$

(ii) At the outer edge of the boundary layer,

$$
\left.\begin{array}{c}
u^{*}\left(x^{*}, \infty\right)=u_{\infty}^{*}, \quad T^{*}\left(x^{*}, \infty\right)=T_{\infty}^{*}, \\
u_{\mathbf{p}}^{*}\left(x^{*}, \infty\right)=u_{\mathrm{p} \infty}^{*}, \quad T_{\mathrm{p}}^{*}\left(x^{*}, \infty\right)=T_{p \infty}^{*} \\
\rho_{\mathrm{p}}^{*}\left(x^{*}, \infty\right)=\rho_{\mathrm{p} \infty}^{*} .
\end{array}\right\}
$$

For the flat-plate problem, it may be assumed that the particle phase is in equilibrium with the gas phase in the external free stream, since there is no pressure gradient. Thus,

$$
u_{\mathrm{p} \infty}^{*}=u_{\infty}^{*}, \quad T_{\mathrm{p} \infty}^{*}=T_{\infty}^{*}, \quad \rho_{\mathrm{p} \infty}^{*}=\beta \rho_{\infty}^{*},
$$

where $\beta$ is the mass loading ratio of the particles.

Sometimes it is convenient to introduce the velocity equilibrium length $\lambda_{\infty}^{*}$ as a characteristic scale in two-phase flow problems:

$$
\lambda_{\infty}^{*}=\frac{\rho_{\mathrm{s}} d^{* 2}}{18 \mu_{\infty}^{*}} u_{\infty}^{*} .
$$

According to the order of magnitude of the slip parameter $x^{*} / \lambda_{\infty}^{*}$, the flow field of a dusty-gas boundary layer can be divided into three distinct regions: (i) The near leading-edge region $\left(x^{*} / \lambda_{\infty}^{*} \ll 1\right)$. The particles have no time to adjust to the local gas motion and then there exists a large velocity slip and a temperature jump between the two phases. The flow properties deviate very little from their frozen values. (ii) The transition region $\left(x^{*} / \lambda_{\infty}^{*} \sim 1\right)$. Owing to the gas-particle interaction, significant changes in the flow properties take place. Slip is moderate and the flow is characterized by non-equilibrium. (iii) The far-downstream region $\left(x^{*} / \lambda_{\infty}^{*} \gg 1\right)$. The particles have enough time to alter their state appreciably and achieve quasiequilibrium with the gas. Then the slip is small.

\section{Asymptotic series-expansion solutions}

Physically, the two-phase boundary-layer flows in the two limiting regions are characterized by quasi-frozen and quasi-equilibrium flows, respectively. Mathematically perturbation solutions in powers of the slip parameter $x^{*} / \lambda_{\infty}^{*}$ (for the large-slip limit) or $\lambda_{\infty}^{*} / x^{*}$ (for the small-slip limit) are suggested. Then the boundarylayer partial differential equations reduce to a system of ordinary differential equations which can be solved by many numerical methods. The following is a brief description of the asymptotic analysis used by Wang \& Glass $(1986 a)$. 


\subsection{The large-slip solution $\left(x^{*} / \lambda_{\infty}^{*} \ll 1\right)$}

In this region, the dimensionless variables and transformation functions are defined as

$$
\left.\begin{array}{c}
x=\frac{x^{*}}{\lambda_{\infty}^{*}}, \quad \eta=\left(\frac{\rho_{\infty}^{*} u_{\infty}^{*}}{2 \mu_{\infty}^{*} x^{*}}\right)^{\frac{1}{2}} y^{*}, \quad \mu=\frac{\mu^{*}}{\mu_{\infty}^{*}}, \quad u=\frac{u^{*}}{u_{\infty}^{*}}, \\
v=\left(\frac{2 \rho_{\infty}^{*} x^{*}}{\mu_{\infty}^{*} u_{\infty}^{*}}\right)^{\frac{1}{2}} v^{*}, \quad f=\eta u-v, \quad T=\frac{T^{*}}{T_{\infty}^{*}}, \\
\rho_{\mathrm{p}}^{*} u_{\mathrm{p}}^{*}=\rho_{\mathrm{p} \infty}^{*} \frac{\partial \psi_{\mathrm{p}}^{*}}{\partial y^{*}}, \quad \rho_{\mathrm{p}}^{*} v_{\mathrm{p}}^{*}=-\rho_{\mathrm{p} \infty}^{*} \frac{\partial \psi_{\mathrm{p}}^{*}}{\partial x^{*}}, \quad f_{\mathrm{p}}=\left(\frac{\rho_{\infty}^{*}}{2 \mu_{\infty}^{*} u_{\infty}^{*} x^{*}}\right)^{\frac{1}{2}} \psi_{\mathrm{p}}^{*} \\
T_{\mathrm{p}}=\frac{T_{\mathrm{p}}^{*}}{T_{\infty}^{*}}, \quad \rho_{\mathrm{p}}=\frac{\rho_{\mathrm{p}}^{*}}{\rho_{\mathrm{p} \infty}^{*}} .
\end{array}\right\}
$$

The following expansion in terms of $x^{*} / \lambda_{\infty}^{*}$ is used:

$$
W(x, \eta)=W^{(0)}(\eta)+x W^{(1)}(\eta)+\ldots,
$$

where the function $W(x, \eta)$ stands for the flow variables $f, u, T, f_{\mathrm{p}}, T_{\mathrm{p}}, \rho_{\mathrm{p}}$ and the superscripts (0) and (1) refer to the zeroth- and first-order approximations, respectively.

Substituting (17)-(18) into the basic equations (1)-(8), the zeroth-, first- or any higher-order equations can be obtained by equating coefficients of $x^{n}(n=0,1, \ldots)$. In this way, the zeroth-order equations become

$$
\begin{gathered}
f^{(0)^{\prime}}-\frac{T^{(0)^{\prime}}}{T^{(0)}} f^{(0)}-u^{(0)}=0 \\
u^{(0)^{\prime \prime}}+\left(\frac{f^{(0)}}{T^{(0)^{\omega+1}}}+\omega \frac{T^{(0)^{\prime}}}{T^{(0)}}\right) u^{(0)^{\prime}}=0 \\
T^{(0)^{\prime \prime}}+\left(\operatorname{Pr} \frac{\left.f^{(0)} \frac{T^{(0)}}{T^{(0)^{\omega+1}}}+\omega \frac{T^{(0)}}{T^{(0)}}\right) T^{(0)^{\prime}}+\operatorname{Ec} \operatorname{Pr}\left(u^{(0)^{\prime}}\right)^{2}=0,}{f_{\mathrm{p}}^{(0)}=\eta,}\right. \\
T_{\mathrm{p}}^{(0)}=1, \\
\rho_{\mathrm{p}}^{(0)}=1,
\end{gathered}
$$

where a prime denotes $\partial / \partial \eta$ and $E c=u_{\infty}^{* 2} / c_{\mathrm{p}}^{*} T_{\infty}^{*}$ is the gas Eckert number based on the free-stream temperature. The boundary conditions for the zeroth-order problem are

$$
f^{(0)}(0)=0, \quad u^{(0)}(0)=0, \quad T^{(0)}(0)=T_{\mathrm{w}}, \quad u^{(0)}(\infty)=1, \quad T^{(0)}(\infty)=1 .
$$

The zeroth-order solution represents the frozen flow limit. Equations (19)-(21) indicate that the zeroth-order gas-phase solution is identical with the similarity solution for the pure-gas cases. Equations (22)-(24) imply that the particle motion is uniform across the boundary layer as if in the free stream. In other words, there is no interaction between the gas and particles and they move independently in the frozen limit.

The first-order equations are:

$$
f^{(1)^{\prime}}-\frac{T^{(0)^{\prime}}}{T^{(0)}} f^{(1)}-3 u^{(1)}+\left(2 u^{(0)}+\frac{T^{(0)^{\prime}}}{T^{(0)}} f^{(0)}\right) \frac{T^{(1)}}{T^{(0)}}-\frac{f^{(0)}}{T^{(0)}} T^{(1)^{\prime}}=0
$$




$$
\begin{gathered}
u^{(1)^{\prime \prime}}+\left(\frac{f^{(0)}}{T^{(0)^{\omega+1}}}+\omega \frac{T^{(0)^{\prime}}}{T^{(0)}}\right) u^{(1)^{\prime}}-2 \frac{u^{(0)}}{T^{(0)^{\omega+1}}} u^{(1)}-\left[(\omega+1) \frac{f^{(0)} u^{(0)^{\prime}}}{T^{(0)^{\omega+2}}}+\omega \frac{T^{(0)^{\prime}} u^{(0)^{\prime}}}{T^{(0)^{2}}}\right] \\
\times T^{(1)}+\omega \frac{u^{(0)^{\prime}}}{T^{(0)}} T^{(1)^{\prime}}+\frac{u^{(0)^{\prime}}}{T^{(0)^{\omega+1}}} f^{(1)}=-2 \beta\left(1-u^{(0)}\right) D, \\
T^{(1)^{\prime \prime}}+\left(\operatorname{Pr} \frac{f^{(0)}}{T^{(0)^{\omega+1}}}+2 \omega \frac{T^{(0)}}{T^{(0)}}\right) T^{(1)^{\prime}}-\left[(\omega+1) \operatorname{Pr} \frac{f^{(0)} T^{(0)^{\prime}}}{T^{(0)^{\omega+2}}}+\omega\left(\frac{T^{(0)^{\prime}}}{T^{(0)}}\right)^{2}+2 \operatorname{Pr} \frac{u^{(0)}}{T^{(0)^{\omega+1}}}\right] \\
\times T^{(1)}+2 E c \operatorname{Pr} u^{(0)^{\prime}} u^{(1)^{\prime}}+\operatorname{Pr} \frac{T^{(0)^{\prime}}}{T^{(0)^{\omega+1}}} f^{(1)}=-2 \beta E c \operatorname{Pr}\left(1-u^{(0)}\right)^{2} D-\frac{2}{3} \beta\left(1-T^{(0)}\right) N u, \\
\eta^{2} f_{\mathrm{p}}^{(1)^{\prime \prime}}-3 \eta f_{\mathrm{p}}^{(1)^{\prime}}+3 f_{\mathrm{p}}^{(1)}=-2 T^{(0)^{\omega}}\left(\eta u^{(0)}-f^{(0)}\right) D, \\
\eta T_{\mathrm{p}}^{(1)^{\prime}}-2 T_{\mathrm{p}}^{(1)}=-\frac{2 \alpha}{3 \operatorname{Pr}^{(0)^{\omega}}\left(T^{(0)}-1\right) N u,} \\
\eta^{2} \rho_{\mathrm{p}}^{(1)^{\prime}}-2 \eta \rho_{\mathrm{p}}^{(1)}=\eta f_{\mathrm{p}}^{(1)^{\prime}}-3 f_{\mathrm{p}}^{(1)}-2 T^{(0)^{\omega}}\left(\eta-f^{(0)}\right) D,
\end{gathered}
$$

where $\alpha=c_{\mathrm{p}}^{*} / c_{s}^{*}$ is the ratio of specific heats of the two phases. In the interaction terms of (27)-(31), D and $N u$ are replaced by their zeroth-order approximations. The corresponding boundary conditions are:

$$
\left.\begin{array}{c}
f^{(1)}(0)=0, \quad u^{(1)}(0)=0, \quad T^{(1)}(0)=0, \quad f_{\mathrm{p}}^{(1)}(0)=0, \\
\mathrm{u}^{(1)}(\infty)=0, \quad T^{(1)}(\infty)=0, \quad f_{\mathrm{p}}^{(1)^{\prime}}(\infty)=0, \quad T_{\mathrm{p}}^{(1)}(\infty)=0, \\
\rho_{\mathrm{p}}^{(1)}(\infty)=0 .
\end{array}\right\}
$$

As shown above, the gas-particle interaction terms do not appear in the zeroth-order equations but just in the first-order equations. It means that the influence of particles is prevalent only in the first- or higher-order terms. This is a major feature of two-phase boundary-layer flows in the large-slip region. Equations (19)-(21) consist of a two-point boundary-value problem, as well as (26)-(28). They are solved numerically by Gear's method (Gear 1971). The solution to (29)-(31) is obtained by numerical integration (Wang \& Glass 1986a).

\subsection{The small-slip solution $\left(\lambda_{\infty}^{*} / x^{*} \ll 1\right)$}

In this region, it is of great advantage to introduce the following slip quantities:

$$
u_{\mathrm{s}}^{*}=u_{\mathrm{p}}^{*}-u^{*}, \quad v_{\mathrm{s}}^{*}=v_{\mathrm{p}}^{*}-v^{*}, \quad T_{\mathrm{s}}^{*}=T_{\mathrm{p}}^{*}-T^{*} .
$$

The dimensionless variables and transformation functions are defined as

$$
\left.\begin{array}{c}
x=\frac{x^{*}}{\lambda_{\infty}^{*}}, \quad \eta=\left(\frac{(1+\beta) \rho_{\infty}^{*} u_{\infty}^{*}}{2 \mu_{\infty}^{*} x^{*}}\right)^{\frac{1}{2}} y^{*}, \quad \mu=\frac{\mu^{*}}{\mu_{\infty}^{*}}, \\
u=\frac{u^{*}}{u_{\infty}^{*}}, \quad v=\left(\frac{2(1+\beta) \rho_{\infty}^{*} x^{*}}{\mu_{\infty}^{*} u_{\infty}^{*}}\right)^{\frac{1}{2}} v^{*}, \quad f=\eta u-v, \quad T=\frac{T^{*}}{T_{\infty}^{*}}, \\
u_{\mathrm{s}}=\frac{u_{\mathrm{s}}^{*}}{u_{\infty}^{*}}, \quad v_{\mathrm{s}}=\left(\frac{2(1+\beta) \rho_{\infty}^{*} x^{*}}{\mu_{\infty}^{*} u_{\infty}^{*}}\right)^{\frac{1}{2}} v_{\mathrm{s}}^{*}, \quad T_{\mathrm{s}}=\frac{T_{\mathrm{s}}^{*}}{T_{\infty}^{*}}, \quad \rho_{\mathrm{p}}=\frac{\rho_{\mathrm{p}}^{*}}{\rho_{\mathrm{p} \infty}^{*}}
\end{array}\right\}
$$

The expansion in terms of $\lambda_{\infty}^{*} / x^{*}$ is

$$
W(x, \eta)=W^{(0)}(\eta)+\frac{1}{x} W^{(1)}(\eta)+\ldots
$$


Similarly, the function $W(x, \eta)$ denotes the flow variables $f, u, T, u_{\mathrm{s}}, v_{\mathrm{s}}, T_{\mathrm{s}}$ and $\rho_{\mathrm{p}}$. In the expansion expression for $u_{\mathrm{s}}, v_{\mathrm{s}}$ and $T_{\mathrm{s}}$, the zeroth-order terms disappear since the slip quantities are small parameters of first order. to

Using (34)-(35) in the basic equations (1)-(8), the zeroth-order problem reduces

$$
\begin{gathered}
f^{(0)^{\prime}}-\frac{T^{(0)^{\prime}}}{T^{(0)}} f^{(0)}-u^{(0)}=0, \\
u^{(0)^{\prime \prime}}+\left(\frac{f^{(0)}}{T^{(0)^{\omega+1}}}+\omega \frac{T^{(0)^{\prime}}}{T^{(0)}}\right) u^{(0)^{\prime}}=0, \\
T^{(0)^{\prime \prime}}+\left(\operatorname{Pr} \frac{1+\beta / \alpha}{1+\beta} \frac{f^{(0)}}{T^{(0)^{\omega+1}}}+\omega \frac{T^{(0)^{\prime}}}{T^{(0)}}\right) T^{(0)^{\prime}}+\operatorname{Ec} \operatorname{Pr}\left(u^{\left\langle()^{\prime}\right.}\right)^{2}=0, \\
\rho_{\mathrm{p}}^{(0)}=\frac{1}{T^{(0)}},
\end{gathered}
$$

with the boundary conditions

$$
f^{(0)}(0)=0, \quad u^{(0)}(0)=0, \quad T^{(0)}(0)=T_{\mathrm{w}}, \quad u^{(0)}(\infty)=1, \quad T^{(0)}(\infty)=1 .
$$

The zeroth-order solution represents the equilibrium-flow limit. Equations (36)-(38) show that the gas-particle mixture behaves like a perfect gas with the following modified properties:

$$
\left.\begin{array}{c}
\bar{\rho}^{*}=(1+\beta) \rho^{*}, \quad \bar{\mu}^{*}=\mu^{*}, \quad \bar{R}^{*}=\frac{1}{1+\beta} R^{*}, \quad \bar{c}_{\mathrm{p}}^{*}=\frac{1+\beta / \alpha}{1+\beta} c_{\mathrm{p}}^{*} \\
\overline{\operatorname{Pr}}=\frac{1+\beta / \alpha}{1+\beta} \operatorname{Pr}, \quad \overline{E c}=\frac{1+\beta}{1+\beta / \alpha} E c,
\end{array}\right\}
$$

where the upper bar denotes the modified quantities. The solution to $(36)-(38)$ is identical to that of (19)-(21) provided the parameters $P r$ and $E c$ in (21) are replaced by $\overline{P r}$ and $\overline{E c}$. In addition, (39) indicates that in the zeroth-order approximation, the dimensionless density of the particle phase is equal to that of the gas in the boundary layer. This means that a constant loading ratio of the particles holds at all points of the boundary layer. Physically, it implies that the particles are 'fixed' to the mass of the gas in which they were originally located and move together with the gas.

For the first-order problem, the slip quantities are given by

$$
\begin{gathered}
u_{\mathrm{s}}^{(1)}=\frac{1}{2 T^{(0)^{\omega}}} f^{(0)} u^{(0)^{\prime}} \\
v_{\mathrm{s}}^{(1)}=\frac{1}{2 T^{(0)^{\omega}}}\left[\eta u^{(0)^{2}}+\eta f^{(0)} u^{(0)^{\prime}}-f^{(0)} u^{(0)}-\frac{T^{(0)^{\prime}}}{T^{(0)}} f^{(0)^{2}}\right] \\
T_{\mathrm{s}}^{(1)}=\frac{3 P r}{4 \alpha T^{(0)^{\omega}}} f^{(0)} T^{(0)^{\prime}}
\end{gathered}
$$

They are readily calculated from the zeroth-order solution.

\section{Finite-difference solution}

The nonlinear partial differential equations $(1)-(8)$, with the boundary conditions of a Dirichlet type (13)-(14), can be solved numerically by a finite-difference procedure. Finite-difference methods of solution for pure-gas boundary-layer equations have been studied for many years (Blottner 1970). In the dusty-gas case, however, some difficulties arise when the finite-difference procedure is applied. First, 
the gas-particle interaction terms are involved in the conservation equations for the gas phase. They appear in the basic equations as a source term. If the treatment of these source terms is not proper, instabilities and physically unrealistic solutions may arise (Patankar 1980). Secondly, an extra set of conservation equations for the particle phase is included. The partial differential equations for the gas are of second order with two boundary conditions at the wall and the outer edge, while the partial differential equations for the particles are of first order with one boundary condition at the wall or the outer edge. Thus a new difference scheme should be constructed for the particle phase. Thirdly, there is no corresponding state equation for the particle phase since the particle phase has no analogue of static pressure. In order to close the system of the governing equations, the $y$-momentum equation for the particles cannot be omitted as for the gas. Thus, it is impossible to take advantage of applying some useful transformation, such as the Howarth-Dorodnitsyn relation, to stretch the normal coordinate $y$ (Flügge-Lotz \& Blottner 1962). Finally, the flow properties of the two-phase boundary layer show quite different features in the three flow regimes. The frozen, non-equilibrium and equilibrium conditions are all encountered when the computation proceeds downstream. Each of these three flow regimes presents its own peculiar problems as far as the calculations are concerned. Therefore, great care must be taken to ensure that a stable and convergent difference solution can be obtained.

The following non-dimensional variables are introduced in order to bring all quantities to the same order of magnitude:

$$
\left.\begin{array}{c}
x=\frac{x^{*}}{\lambda_{\infty}^{*}}, \quad y=\frac{y^{*}}{\lambda_{\infty}^{*}} \operatorname{Re}_{\infty}^{\frac{1}{2}}, \quad \mu=\frac{\mu^{*}}{\mu_{\infty}^{*}}, \\
u=\frac{u^{*}}{u_{\infty}^{*}}, \quad v=\frac{v^{*}}{u_{\infty}^{*}} R_{\infty}^{\frac{1}{2}}, \quad T=\frac{T^{*}}{T_{\infty}^{*}}, \quad \rho=\frac{\rho^{*}}{\rho_{\infty}^{*}}, \\
u_{\mathrm{p}}=\frac{u_{\mathrm{p}}^{*}}{u_{\infty}^{*}}, \quad v_{\mathrm{p}}=\frac{v_{\mathrm{p}}^{*}}{u_{\infty}^{*}} \operatorname{Re}_{\infty}^{\frac{1}{2}}, \quad T_{\mathrm{p}}=\frac{T_{\mathrm{p}}^{*}}{T_{\infty}^{*}}, \quad \rho_{p}=\frac{\rho_{\mathrm{p}}^{*}}{\rho_{\infty}^{*}},
\end{array}\right\}
$$

where $R e_{\infty}=\rho_{\infty}^{*} u_{\infty}^{*} \lambda_{\infty}^{*} / \mu_{\infty}^{*}$ is the flow Reynolds number based on $\lambda_{\infty}^{*}$. Then, the governing equations (1)-(8) reduce to non-dimensional form as

$$
\begin{gathered}
\frac{\partial}{\partial x} \rho u+\frac{\partial}{\partial y} \rho v=0 \\
\rho\left(u \frac{\partial u}{\partial x}+v \frac{\partial u}{\partial y}\right)=\mu \frac{\partial^{2} u}{\partial y^{2}}+\left(\frac{\mathrm{d} \mu}{\mathrm{d} T} \frac{\partial T}{\partial y}\right) \frac{\partial u}{\partial y}+\rho_{\mathrm{p}}\left(u_{\mathrm{p}}-u\right) \mu D \\
\rho\left(u \frac{\partial T}{\partial x}+v \frac{\partial T}{\partial y}\right)=\frac{\mu}{\operatorname{Pr}} \frac{\partial^{2} T}{\partial y^{2}}+\frac{1}{\operatorname{Pr}} \frac{\mathrm{d} \mu}{\mathrm{d} T}\left(\frac{\partial T}{\partial y}\right)^{2}+E c \mu\left(\frac{\partial u}{\partial y}\right)^{2} \\
+E c \rho_{\mathrm{p}}\left[\left(u_{\mathrm{p}}-u\right)^{2}+\frac{1}{R e_{\infty}}\left(v_{\mathrm{p}}-v\right)^{2}\right] \mu D+\frac{1}{3 \operatorname{Pr}} \rho_{\mathrm{p}}\left(T_{\mathrm{p}}-T\right) \mu N u \\
\rho=\frac{1}{T} \\
\frac{\partial}{\partial x} \rho_{\mathrm{p}} u_{\mathrm{p}}+\frac{\partial}{\partial y} \rho_{\mathrm{p}} v_{\mathrm{p}}=0 \\
u_{\mathrm{p}} \frac{\partial u_{\mathrm{p}}}{\partial x}+v_{\mathrm{p}} \frac{\partial u_{\mathrm{p}}}{\partial y}=-\left(u_{\mathrm{p}}-u\right) \mu D
\end{gathered}
$$




$$
\begin{gathered}
u_{\mathrm{p}} \frac{\partial v_{\mathrm{p}}}{\partial x}+v_{\mathrm{p}} \frac{\partial v_{\mathrm{p}}}{\partial y}=-\left(v_{\mathrm{p}}-v\right) \mu D \\
u_{\mathrm{p}} \frac{\partial T_{\mathrm{p}}}{\partial x}+v_{\mathrm{p}} \frac{\partial T_{\mathrm{p}}}{\partial y}=-\frac{\alpha}{3 P r}\left(T_{\mathrm{p}}-T\right) \mu N u .
\end{gathered}
$$

The non-dimensional form of the boundary conditions (13)-(14) are

and

$$
u(x, 0)=0, \quad v(x, 0)=0, \quad T(x, 0)=T_{\mathrm{w}}, \quad v_{\mathrm{p}}(x, 0)=0,
$$

$$
\left.\begin{array}{c}
u(x, \infty)=1, \quad T(x, \infty)=1, \\
u_{\mathrm{p}}(x, \infty)=1, \quad T_{\mathrm{p}}(x, \infty)=1, \quad \rho_{\mathrm{p}}(x, \infty)=\beta
\end{array}\right\}
$$

In differencing the above partial differential equations (44)-(51), the derivatives in the $x$-direction are replaced by a backward difference and then the difference scheme is implicit. For the $x$-momentum and energy equations of the gas, (45)-(46), a sixpoint scheme is used and the finite-difference equations become simultaneous linear algebraic equations with a tridiagonal coefficient matrix :

$$
A_{n} W_{m+1, n+1}+B_{n} W_{m+1, n}+C_{n} W_{m+1, n-1}=D_{n},
$$

where $W=u$ and $T$, and $A_{n}, B_{n}, C_{n}$ and $D_{n}$ are the matrix elements while $m, n$ are the indices of the grid point considered (figure 2). This tridiagonal-matrix system can be solved by the well-known Thomas algorithm (Anderson, Tannehill \& Pletcher 1984). A four-point scheme with a central-difference formula in the $y$-direction is used for the gas continuity equation and another four-point scheme with a backward-difference formula in the $y$-direction is used for all the equations of the particles. The resulting difference equations are

$$
A_{n} W_{m+1, n \pm 1}+B_{n} W_{m+1, n}=C_{n}
$$

where $W=u_{\mathrm{p}}, v_{\mathrm{p}}, T_{\mathrm{p}}, \rho_{\mathrm{p}}$ and $\rho v$ ( $\rho v$ is employed as the dependent variable when solving the gas continuity equation). It is clear that the system of algebraic equations $(55)$ is readily solved provided the boundary conditions are specified.

It should be pointed out that along the plate there is a critical point where the particle velocity at the wall becomes zero and reaches equilibrium with the gas at the wall. As the particle velocity vanishes, the particles tend to accumulate at the wall and the density of the particles near the wall may become very large. The discussion of a dense gas-particle system, however, is beyond the subject of this paper. Then it is assumed that Brownian diffusion process in the near-wall region prevents the formation of a dense bed of particles (Soo 1967, 1968). When the particles are small, it is a reasonable assumption and can be verified by the small-slip asymptotic solution. After the critical point, quite simple compatibility conditions are derived as follows :

$$
u_{\mathrm{p}}(x, 0)=0, \quad T_{\mathrm{p}}(x, 0)=T_{\mathrm{w}},
$$

for $x \geqslant x_{\text {cri }}$ where $x_{\text {cri }}$ is the $x$-coordinate for the critical point. The value of $x_{\text {cri }}$ can be determined by the compatibility equation

$$
\frac{\partial u_{\mathrm{pw}}}{\partial x}=-(\mu D)_{\mathrm{w}}
$$

and the condition $u_{\mathrm{pw}}=0$. The compatibility conditions (56) provide supplemental boundary conditions for the particle velocity and temperature so that the six-point scheme can also be used for the $x$-momentum and energy equations of the particles. 


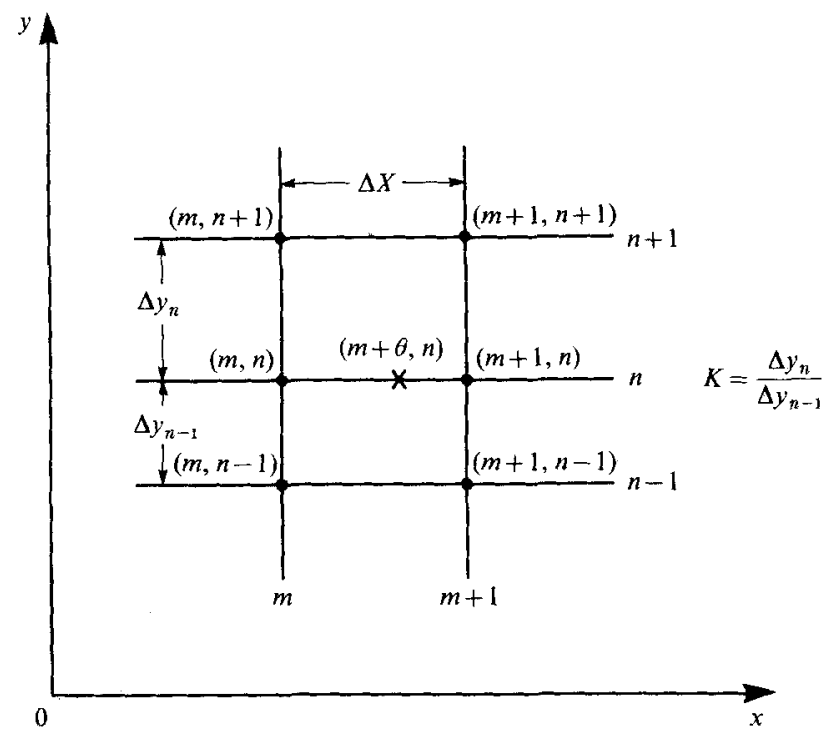

Figure 2. Grid points for the finite-difference schemes.

Thus, the corresponding difference equations have the form of (54). The six-point scheme is more accurate than the four-point scheme.

In order to start the computation, the initial profiles of the flow variables are required at some given point $x_{0}$. In most studies of pure-gas boundary-layer flows, the initial profiles are obtained from the similarity solution. For the dusty-gas case, however, no analogous solutions exist. In this analysis, three different types of initial profiles are employed for comparison: the zeroth-order large-slip solution, the firstorder large-slip solution and the extended Wu-type profiles. The first two solutions can be obtained from the asymptotic analysis. The third one is a modification of the Wu-type initial profiles for the pure-gas case (Wu 1960) and it is given by

$$
\left.\begin{array}{rl}
u(0, y) & =0(\text { for } y=0) \text { or } 1(\text { for } y>0), \quad v(0, y)=0, \\
T(0, y) & =T_{\mathrm{w}}(\text { for } y=0) \text { or } 1(\text { for } y>0), \\
\rho(0, y) & =\frac{1}{T_{\mathrm{w}}}(\text { for } y=0) \text { or } 1(\text { for } y>0), \\
u_{\mathrm{p}}(0, y) & =1, \quad v_{\mathrm{p}}(0, y)=0, \quad T_{\mathrm{p}}(0, y)=1, \quad \rho_{\mathrm{p}}(0, y)=\beta .
\end{array}\right\}
$$

The details of the finite-difference procedure used in this paper are described by Wang \& Glass (1986b).

\section{Results and discussions}

Both the asymptotic and finite-difference solutions were obtained numerically. In the computation, the values of some parameters were chosen as $\operatorname{Pr}=1.0, E c=1.0$, $\alpha=1.0, \beta=1.0$ and $T_{\mathrm{w}}=0.5$, for example.

The numerical results for the Stokes case are presented first. To illustrate the effect of the gas-viscosity index on the flow structure, figure 3 shows the zeroth-and firstorder velocities for the gas in the large-slip region and the first-order slip velocities 


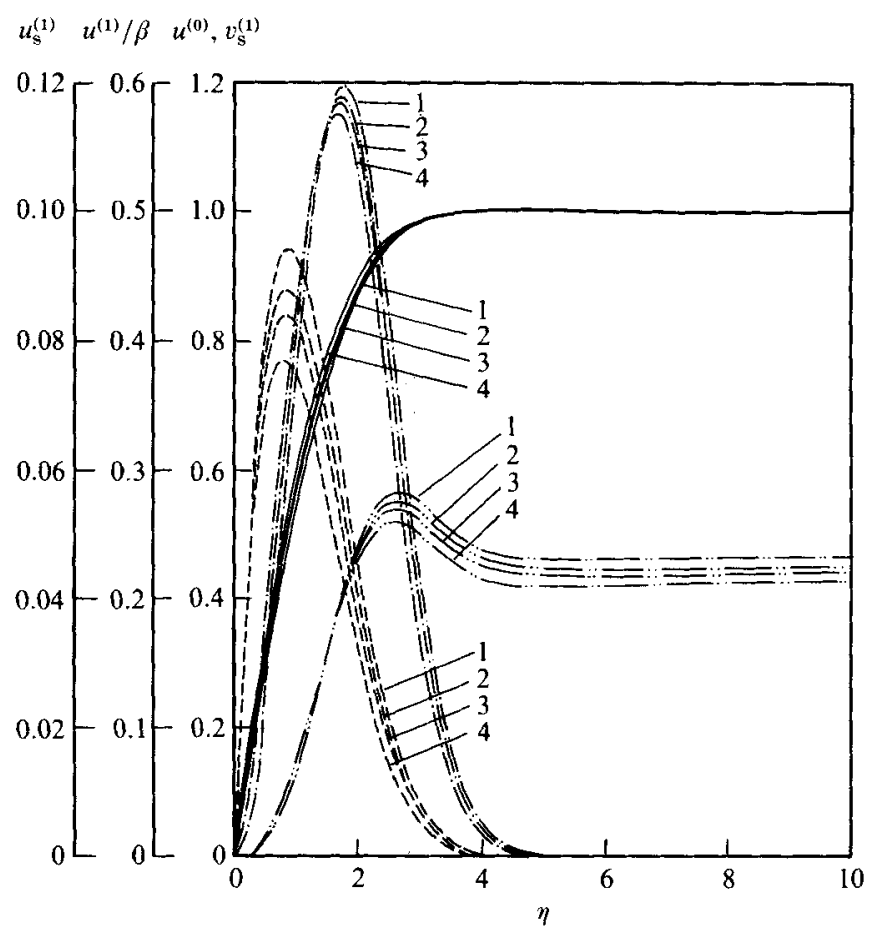

Frgure 3. Zeroth-order and first-order velocities in the limiting regions, --, $u^{(0)} ;---, u^{(1)} / \beta$; $\ldots, u_{\mathrm{s}}^{(1)} ; \cdots \cdots, v_{\mathrm{s}}^{(1)} .1, \omega=0.50 ; 2, \omega=0.67 ; 3, \omega=0.77 ; 4, \omega=1.00$.

in the small-slip region, which are the asymptotic solutions. The finite-difference solution is introduced in figures 4 to 6 , where the profiles of the velocity components and temperature for the two phases at three typical distances, $x=0.105,1.05$ and 10.05 , are given, respectively. In the finite-difference procedure, the grid parameters are $\Delta x=0.001, \Delta y_{1}=0.03, K=1.05$ and $\theta=0.75$ while the initial profiles are the first-order asymptotic solution. It is noted that, at $x=0.105$ of the large-slip region (see figure 4), there are very large differences between the flow profiles for the two phases. The gas adjusts its velocity and temperature at the wall to meet the conditions of no slip in velocity and no jump in temperature as in the case of a puregas boundary-layer flow. The particles, however, cannot respond to the abrupt changes and tend to keep their values in the original uniform motion. Then the flow is quasi-frozen. With the variable transformation $y=(2 x)^{\frac{1}{2}} \eta$, the asymptotic largeslip solution at the position $x=0.105$ is shown in figure 4 , for comparison. The agreement between the two solutions is excellent and it indicates that the asymptotic solution for the large-slip limit is valid until $x=0.1$. With increasing $x$, the interaction between the gas and particles causes the particles to be decelerated and cooled in the cold-wall case while the gas is accelerated and heated. In the transition region $(x \sim 1)$, the changes in the two-phase flow properties to the frozen limit are considerable although the velocity slip and temperature defect between the gas and particles are still fairly large. It can be seen in figure 5, where the distribution of flow variables at $x=1.05$ is given. The two-phase boundary-layer flow is characterized by non-equilibrium in this region. In figure 6 , the flow structure in the small-slip region $(x=10.05)$ indicates that all the flow variables are almost the same for the two phases. In this far-downstream region, the particles have enough time to approach 


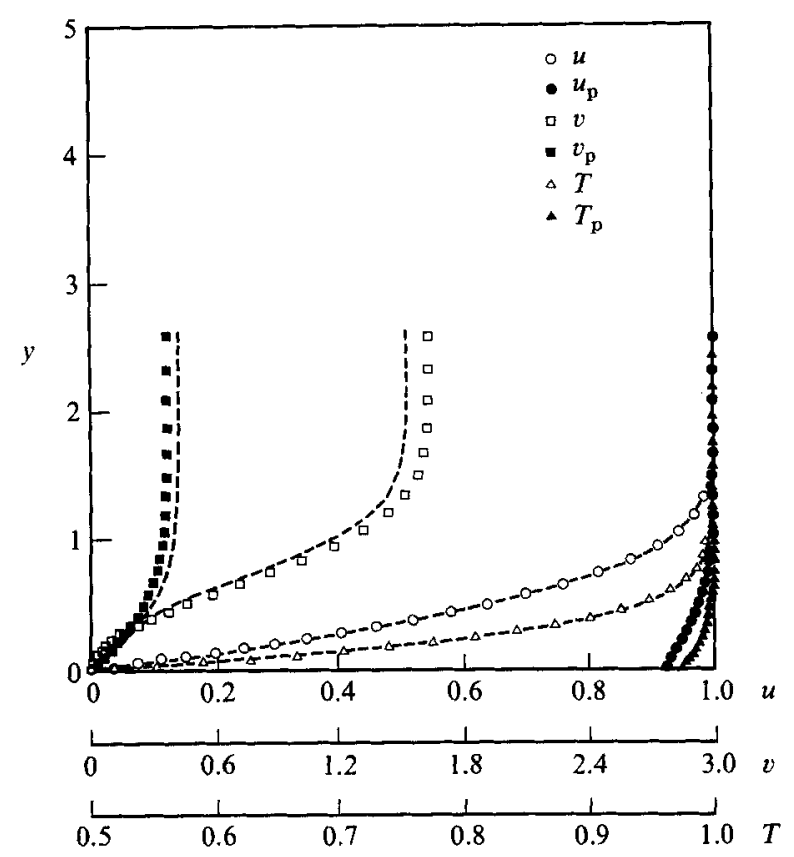

FigURE 4. Flow profiles $u, v$ and $T$ vs. $y$ in the large-slip region $(x=0.105)$. --- , asymptotic solution.

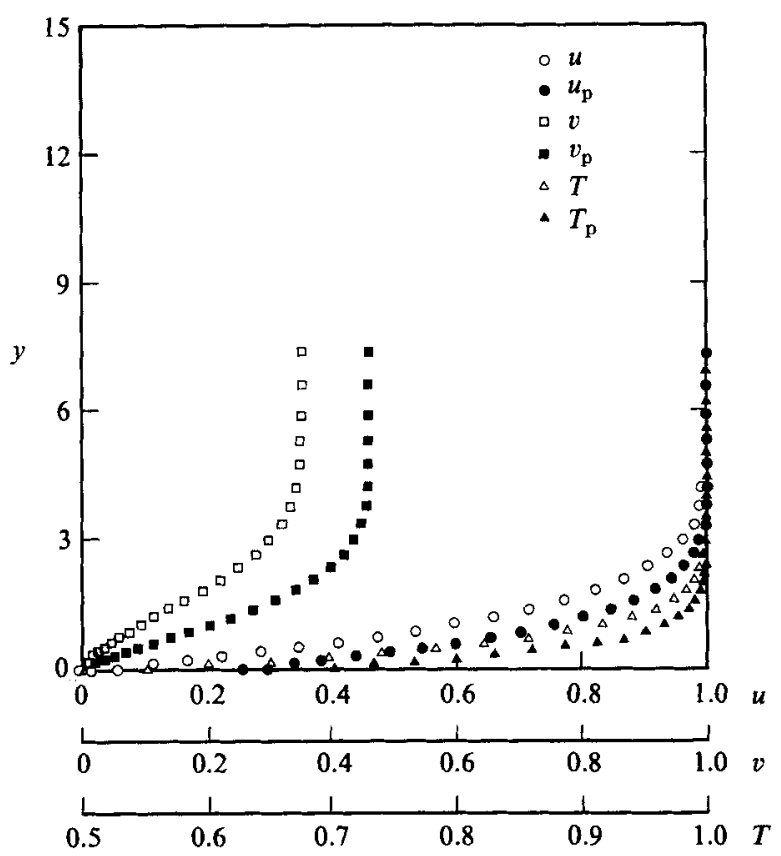

Ftaure 5. Flow profiles $u, v$ and $T v s . y$ in the transition region $(x=1.05)$. 


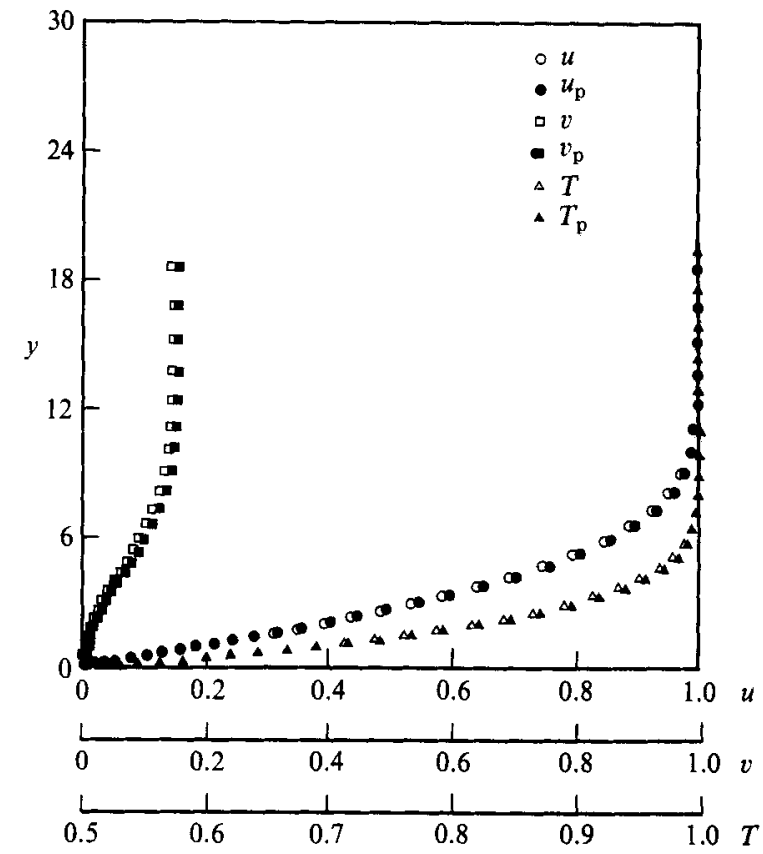

Frgure 6. Flow profiles $u, v$ and $T$ vs. $y$ in the small slip region $(x=10.05)$.

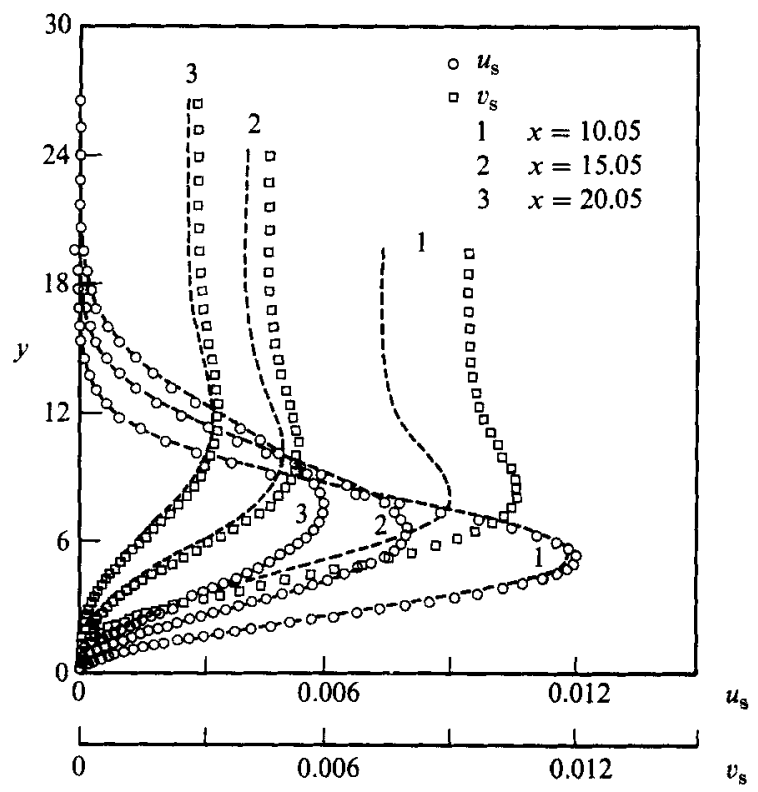

Figure 7. Slip velocities $u_{\mathrm{s}}$ and $v_{\mathrm{p}} v s . y$ in the small-slip region. -----, asymptotic solution.

the state of the gas motion and then a quasi-equilibrium flow is achieved. The asymptotic and finite-difference results for the velocities in the small-slip region are compared at three positions $(x=10.05,15.05$ and 20.05) in figure 7 . For increased clarity, the slip quantities are employed. The variable transformation here is $y=$ $(2 x /(1+\beta))^{\frac{1}{2}} \eta$. Similarly, the agreement is very good and it indicates that, in practice, the asymptotic solution for the small-slip limit can be applied with adequate accuracy when $x \geqslant 10$. 


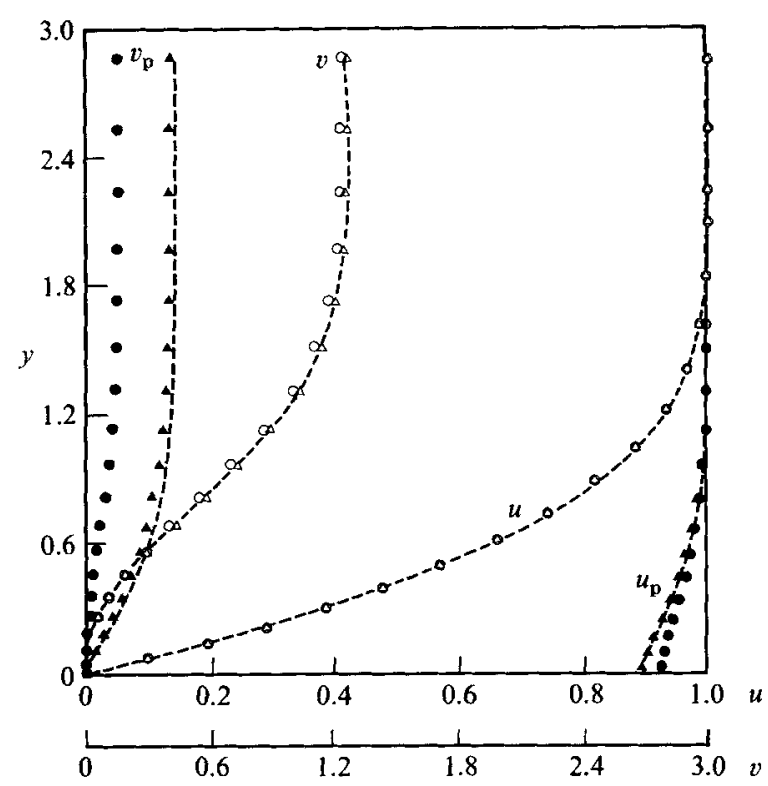

Figure 8. Comparison of velocity profiles with different initial profiles at $x=1.05$. $\triangle$, extended Wu-type; $\mathrm{O}$, zeroth order; ---, first order.

Finite-difference calculations were done with the three types of initial profiles as mentioned before. The extended $\mathrm{Wu}$-type profiles apply at the leading edge and the computation can be started at $x_{0}=0$. With the two asymptotic-solution profiles, the computation began at $x_{0}=0.05$ in this analysis, since there is a singularity at the leading edge. The numerical results for the three types of initial profiles were obtained along the entire length of the flat plate. It is shown from the comparison that, for the gas phase, the resulting flow profiles with these three initial profiles are very similar to each other. For the particle phase the same conclusion can be reached for the first-order and extended Wu-type profiles. By contrast, some differences in the particle flow properties can be found between the cases of the zeroth-order asymptotic profiles and the other two profiles, especially in the large-slip region (see figure 8). This phenomenon can be explained as follows. The zeroth-order profiles assume that, at some distance after the leading edge, the particles still possess their original uniform.state of motion. It is not true physically since only at the leading edge are the particle flow profiles really uniform as assumed in the extended Wu-type profiles. But the influence of the initial profiles is gradually damped out with increasing $x$ and the same flow structure can be obtained from the three initial profiles in the far-downstream region. It is very useful to point out that the finitedifference computation with the extended Wu-type of initial profiles yields a reasonable solution compared with the first-order asymptotic profiles. It leads to a great simplification since it is not necessary to solve the asymptotic solution in the large-slip region and the finite-difference computation can be readily started at the leading edge. This way of presenting the initial conditions is advantageous for some other problems, for example, in the study of the sidewall boundary layer induced by a moving shock wave in a dusty-gas shock tube (Wang \& Glass $1986 \mathrm{c}$ ).

For the first time, the numerical results in the case of a non-Stokes relation for the interaction terms are presented, which are very important for high-velocity flows. In this computation, the particle Reynolds number $R e_{\mathrm{p}}=\rho_{\infty}^{*} u_{\infty}^{*} d^{*} / \mu_{\infty}^{*}$ is assumed to be 


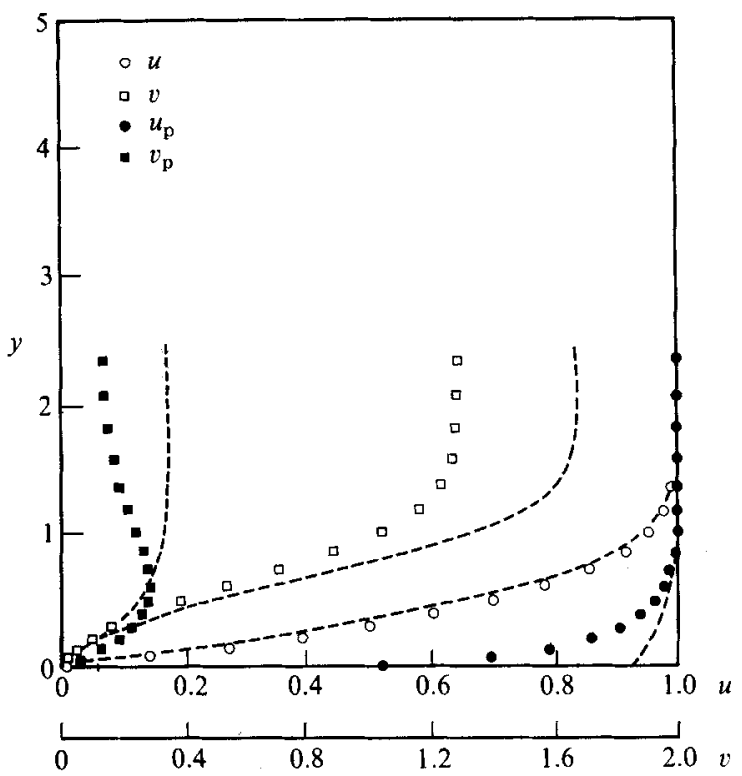

Frgure 9. Flow velocities $u, v$ vs. $y$ in the non-Stokes case at $x=0.1,----$, , Stokes case.

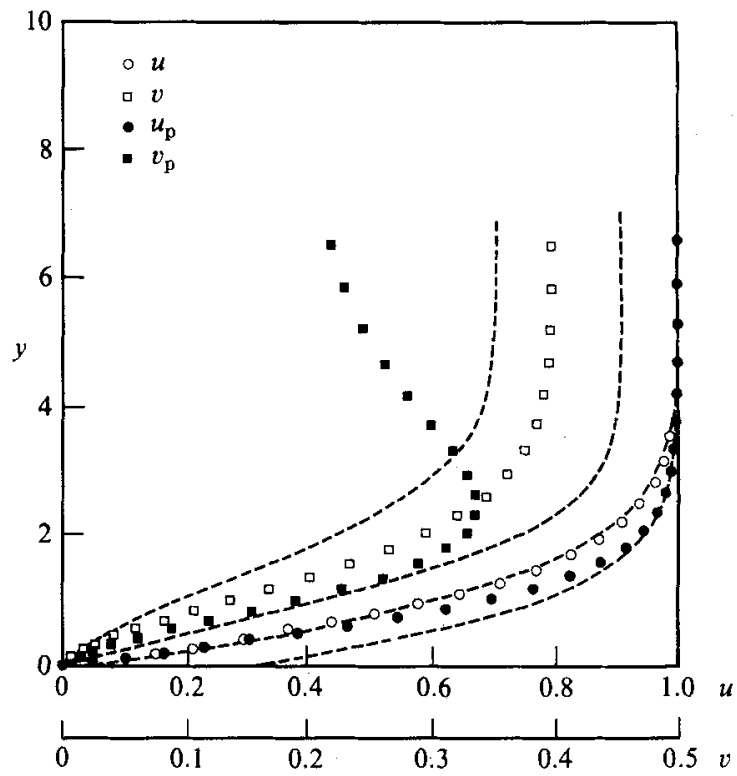

Figure 10. Flow velocities in the non-Stokes case at $x=1.0,----$, Stokes case.

equal to 100 . The velocity profiles for the two phases in the three flow regimes (at $x=0.1,1.0$ and 5.0) are shown in figures 9-11, where corresponding results in the Stokes case are given for comparison. It is seen that in the large-slip and transition regions, quite large differences in the particle flow properties appear between the two cases, especially in the region near the wall. In the non-Stokes case, the particles change their velocity and temperature more quickly and the relaxation process takes place over a distance shorter than $\lambda_{\infty}^{*}$, which is the characteristic length for the relaxation process in the Stokes case. The Stokes relation underestimates the 


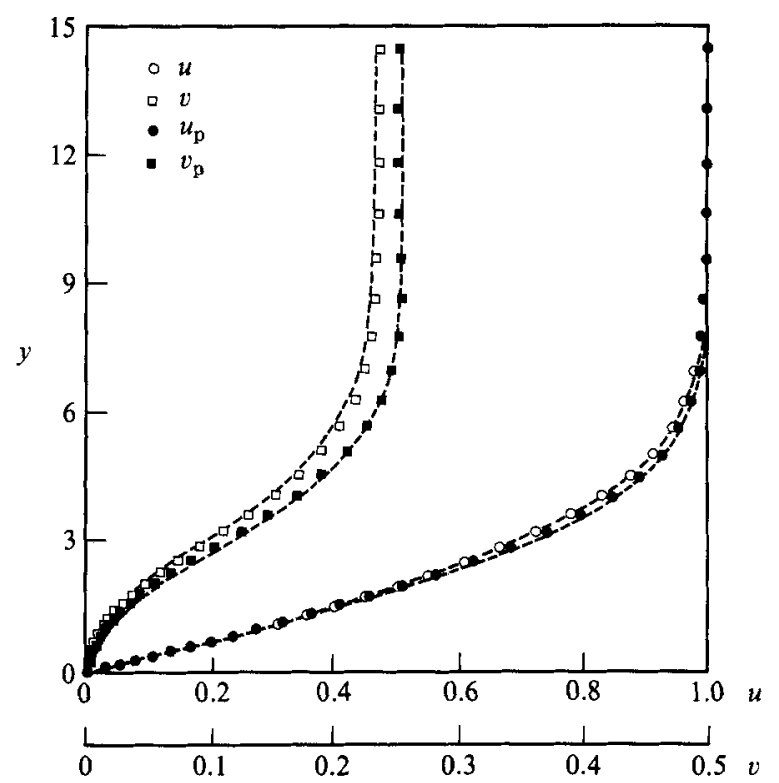

Figure 11. Flow velocities in the non-Stokes case at $x=5.0,----$, Stokes case.

interaction terms when the slip Reynolds number or the particle Reynolds number is large. When approaching the wall across the boundary layer, the slip Reynolds number increases from zero at the outer edge to its maximum value at the wall so that the largest changes between the two interaction relations appear at the wall. In addition, it is known that the interaction with the gas is the only controlling mechanism for the particle motion but the interaction with the particles is just a secondary mechanism (compared with the viscous effect) for the gas motion. As a result, the changes in the particle flow properties are much greater than those in the gas flow properties. However, in the far-downstream region, the flow profiles obtained from the two interaction relations reduce to the same results, since the slip Reynolds numbers there become so small that the two relations give interaction terms of the same magnitude. This fact can be seen in figure 11.

Finally, the characteristic boundary-layer quantities are calculated from the flow profiles of the gas phase. The characteristics of practical interest include the wall shear stress $\tau_{\mathrm{w}}^{*}=\left(\mu^{*} \partial u^{*} / \partial y^{*}\right)_{\mathrm{w}}$, the wall heat-transfer rate $\dot{q}_{\mathrm{w}}^{*}=-\left(k^{*} \partial T^{*} / \partial y^{*}\right)_{\mathrm{w}}$ and the displacement thickness,

$$
\delta^{*}=\int_{0}^{\infty}\left(1-\rho^{*} u^{*} / \rho_{\infty}^{*} u_{\infty}^{*}\right) \mathrm{d} y^{*} .
$$

The non-dimensional characteristics are defined:

$$
\tau_{\mathrm{w}}=\frac{\tau_{\mathrm{w}}^{*}}{\rho_{\infty}^{*} u_{\infty}^{* 2}} R e_{\infty}^{\frac{1}{2}}, \quad \dot{q}_{\mathrm{w}}=\frac{\dot{q}_{\mathrm{w}}^{*}}{\rho_{\infty}^{*} u_{\infty}^{* 3}} R e_{\infty}^{\frac{1}{2}}, \quad \delta=\frac{\delta^{*}}{\lambda_{\infty}^{*}} R e_{\infty}^{\frac{1}{2}} .
$$

Then they can be expressed in terms of the non-dimensional parameters as

$$
\tau_{\mathrm{w}}=\mu_{\mathrm{w}}\left(\frac{\partial u}{\partial y}\right)_{\mathrm{w}}, \quad \dot{q}_{\mathrm{w}}=-\frac{\mu_{\mathrm{w}}}{\operatorname{EcPr}}\left(\frac{\partial T}{\partial y}\right)_{\mathrm{w}}, \quad \delta=\int_{0}^{\infty}(1-\rho u) \mathrm{d} y .
$$




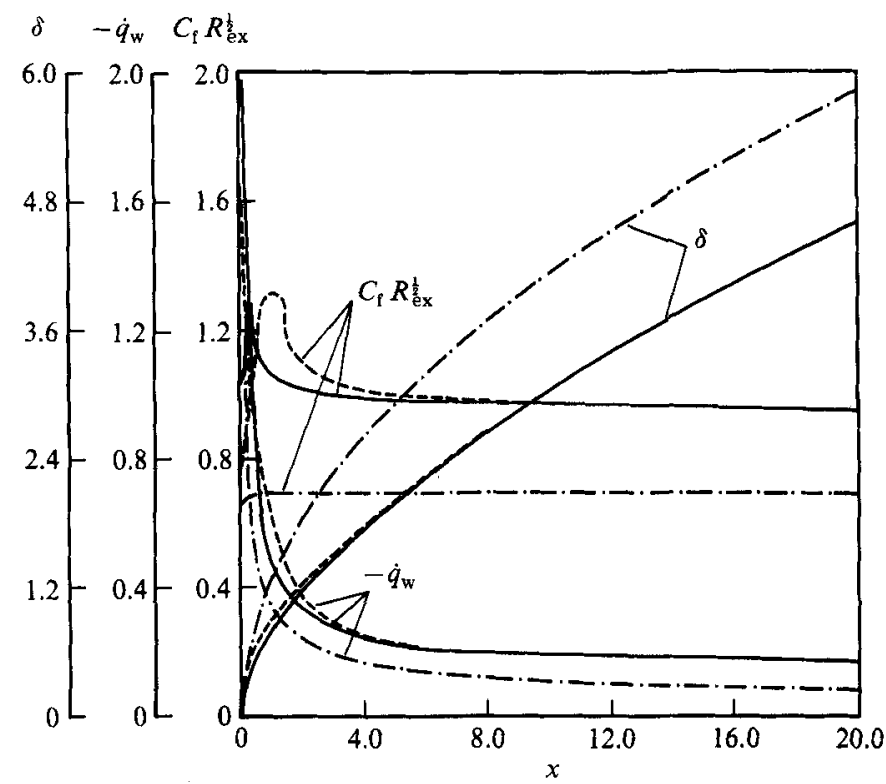

Figure 12. Characteristic boundary-layer quantities along the plate. - - , non-Stokes case; ----, Stokes case; - - - pure-gas case.

Sometimes, another conventional parameter is employed:

$$
c_{f} R e_{x}^{\frac{1}{2}}=2 x^{\frac{1}{2}} \tau_{\mathrm{w}},
$$

where $c_{f}=2 \tau_{\mathrm{w}}^{*} / \rho_{\infty}^{*} u_{\infty}^{* 2}$ is the local skin-friction coefficient and $R e_{x}=\rho_{\infty}^{*} u_{\infty}^{*} x^{*} / \mu_{\infty}^{*}$ is the local Reynolds number. The skin friction, heat-transfer and displacement thickness as a function of the coordinate $x$ are shown in figure 12. For comparison, the non-Stokes solution, the Stokes solution and the pure-gas solution are presented together. It is noted that, owing to the presence of particles, the skin friction coefficient and the wall heat-transfer increase while the displacement thickness decreases. Here the gas gains some kinetic and thermal energy from the particles through the interaction and the gas velocity and temperature increase above the pure-gas case. Consequently, the velocity and temperature gradients for the gas increase at the wall so that the skin friction and heat transfer increase while the velocity reaches its free-stream value more quickly and the boundary-layer thickness is reduced. Concerning the differences between the non-Stokes and Stokes solutions, a similar argument holds. In the near leading-edge region, the interaction in the nonStokes case is usually much greater than that in the Stokes case so that the skin friction and heat transfer for the non-Stokes relation are greater than those for the Stokes relation. On the other hand, the relaxation process in the non-Stokes case takes place much more quickly than that in the Stokes case so that the critical point is reached earlier for the non-Stokes case. In other words, there may be a region where the flow for the non-Stokes case has already become near-equilibrium while the flow in the Stokes case is still in non-equilibrium. In this region the skin friction and wall heat-transfer in the Stokes case are greater than those in the non-Stokes case. As expected, the non-Stokes and Stokes solutions approach the same asymptotic value far downstream. At the critical point, the two-phase system transits from a non-equilibrium flow to an equilibrium flow. Due to this significant 
change in the flow properties, there is an inflexion point along the curve of the characteristic quantities at the position of the critical point.

\section{Concluding remarks}

The properties of a compressible laminar boundary-layer flow of a dusty gas over a semi-infinite flat plate were analysed numerically. The series-expansion method provided the asymptotic solutions in the two limiting regions. The finite-difference method gave the complete description of the flow structure along the whole length of the plate. The agreement between the asymptotic and finite-difference solutions is excellent. The numerical results for the dilute two-phase system show the effects of the gas-particle interactions on the flow properties. These include alterations in the flow profiles, an increase in the skin friction and wall heat-transfer rate and a decrease in the displacement thickness. The comprative studies on the different initial profiles indicate that the finite-difference computation can be started at the leading edge using the extended $W u$-type of initial profiles. The comparative studies on the different interaction relations show that the relevant non-Stokes interaction relation should be included in the case of large-particle Reynolds numbers. Undoubtedly, some improvement can be made in the future by taking into account some other effects such as extra forces (for example, gravity, buoyancy, lift or even electrostatic forces), distribution of particle size and non-spherical shapes.

We wish to express our appreciation to Dr W. S. Liu and Dr J. P. Sislian for their valuable discussions. One of us (B.Y.W.) is grateful to the Institute of Mechanics, Chinese Academy of Sciences, and to UTIAS for making possible her research leave at UTIAS.

The financial assistance received from the Natural Sciences and Engineering Research Council under grant no. A1647, the US Air Force under grant AF-AFOSR87-0124 and the Defence Nuclear Agency under contract DNA 001-85-C-0368 is acknowledged with thanks.

\section{REFERENCES}

Anderson, D. A., TAnnehile, J. C. \& Pretcher, R. H. 1984 Computational Fluid Mechanics and Heat Transfer. Hemisphere.

BlottNer, F. G. 1970 AIAA J. 8, 193-205.

DiGiovannt, P. R. \& Lee, S. L. $1974 J$. Appl. Mech. 41, 35.

FlügGE-LOtz, I. \& BLOTtNer, F. G. 1962 AFOSR 2206.

GEAR, C. W. 1971 Numerical Initial Value Problems in Ordinary Differential Equations. PrenticeHall.

Grlbert, M., Davis, L. \& Altman, D. 1955 Jet Propul. 25, 26.

HaMED, A. \& TABakofF, W. 1973 AIAA paper 73-687.

$\mathrm{J}_{\mathrm{AIN}}$, A. C. \& GHosh, A. 1979 Z. Flugwiss. weltraum forschung 3, 379.

Knudsen, J. G. \& Katz, D. L. 1958 Fluid Mechanics and Heat Transfer. MeGraw-Hill.

Liv, J. T. C. 1967 Astro Acta 13, 369.

MarbLe, F. E. 1963 Combustion and Propulsion, 5th AGARD Colloquium, Oxford. Pergamon.

Osiptsov, A. N. 1980 Fluid Dyn. 15, 512.

Otterman, B. \& Lee, S. L. 1970 Proc. Heat Transfer and Fluid Mechanics Institute, Monterey. Stanford University Press.

Patankar, S. V. 1980 Numerical Heat Transfer and Fluid Flow. Hemisphere.

Prabha, S. \& JaIN, A. C. 1982 Proc. 13th Intl Symp. Space Technology and Science, Tokyo. 
Rudinger, G. 1980 Fundamentals of Gas-Particle Flow. Elsevier.

Singleton, R. E. 1965 Z. angew. Math. Phys. 16, 421.

Soo, S. I. 1967 Fluid Dynamics of Multiphase Systems. Waltham: Blaisdell.

Soo, S. L. 1968 Z. angew. Math. Phys. 19, 545.

TABakoff, W. \& HaMed, A. 1972 AIAA paper $72-87$.

Wang, B. Y. \& Glass, I. I. $1986 a$ UTIAS Rep. 310.

Wang, B. Y. \& Glass, I. I. $1986 b$ UTIAS Rep. 311.

Wang, B. Y. \& Glass, I. I. 1986 c UTIAS Rep. 312.

WU, J. C. 1960 Douglass Aircraft Company Inc. Rep. SM-37484. 